[Article]

\title{
铜物种对 $\mathrm{Cu} / \mathrm{Fe}_{2} \mathrm{O}_{3}$ 水煤气变换反应催化剂性能的影响
}

\author{
林性贻* 马俊涛陈崇启詹瑛瑛郑 起 \\ (福州大学化肥催化剂国家工程研究中心, 福州 350002)
}

\begin{abstract}
摘要: 采用共沉淀法制备了系列铜负载量不同的 $\mathrm{Cu} / \mathrm{Fe}_{2} \mathrm{O}_{3}$ 水煤气变换(WGS)催化剂, 并考察了铜负载量对催 化剂结构和水煤气变换反应性能的影响. 结果表明, $\mathrm{Cu} / \mathrm{Fe}_{2} \mathrm{O}_{3}$ 催化剂呈现出良好的水煤气反应性能, 当 $\mathrm{CuO}$ 质 量分数为 $20 \%$ 时, 催化剂的WGS 性能最优, $250{ }^{\circ} \mathrm{C}$ 时 CO 转化率高达 $97.2 \%$, 同时热稳定性也最好. 运用X射 线粉末衍射 $(X R D) 、 \mathrm{~N}_{2}$ 物理吸脱附和 $\mathrm{H}_{2}$ 程序升温还原 $\left(\mathrm{H}_{2}-\mathrm{TPR}\right)$ 等手段对 $\mathrm{Cu} / \mathrm{Fe}_{2} \mathrm{O}_{3}$ 催化剂的物相、织构特征及还 原性能进行了表征, 结果表明, $\mathrm{CuFe}_{2} \mathrm{O}_{4}$ 物种的存在极大地改善了催化剂的还原性能和WGS 反应活性. 这是由 于 $\mathrm{CuFe}_{2} \mathrm{O}_{4}$ 特殊的尖晶石结构有利于 $\mathrm{Cu}$ 微晶的稳定; 同时, $\mathrm{CuFe}_{2} \mathrm{O}_{4}$ 在低温下即被还原为单质铜, 有利于促进 催化剂体系中电子的转移. 此外, 通过 $\left(\mathrm{NH}_{4}\right)_{2} \mathrm{CO}_{3}$ 溶液处理, 研究了独立相 $\mathrm{CuO}$ 对 $\mathrm{Cu} / \mathrm{Fe}_{2} \mathrm{O}_{3}$ 催化剂WGS 反应 性能的影响, 结果发现, 独立相 $\mathrm{CuO}$ 的存在, 有利于 $\mathrm{H}$ 原子在各组分传递, 从而促进催化剂的 $\mathrm{CuFe}_{2} \mathrm{O}_{4}$ 的还原, 改善 $\mathrm{Cu} / \mathrm{Fe}_{2} \mathrm{O}_{3}$ 催化剂的WGS 反应性能.
\end{abstract}

关键词: 水煤气变换; $\mathrm{Cu} / \mathrm{Fe}_{2} \mathrm{O}_{3}$ 催化剂; 铜负载量; $\mathrm{CuFe}_{2} \mathrm{O}_{4}$

中图分类号: 0643.3

\section{Influence of Copper Species on Performance of $\mathrm{Cu} / \mathrm{Fe}_{2} \mathrm{O}_{3}$ Catalysts for Water Gas Shift Reaction}

\author{
LIN Xing-Yi $\quad$ MA Jun-Tao CHEN Chong-Qi ZHAN Ying-Ying ZHENG Qi \\ (National Engineering Research Center of Chemical Fertilizer Catalyst, Fuzhou University, Fuzhou 350002, P. R. China)
}

\begin{abstract}
A series of $\mathrm{Cu} / \mathrm{Fe}_{2} \mathrm{O}_{3}$ catalysts with different $\mathrm{Cu}$ loadings were prepared using a co-precipitation method, and the relationship between their structures and catalytic activities for the water gas shift (WGS) reaction was carefully examined. It was found that the as-prepared $\mathrm{Cu} / \mathrm{Fe}_{2} \mathrm{O}_{3}$ catalysts exhibit excellent WGS performances, in particular, the one containing $20 \%$ (w) CuO (CF-20) shows the best catalytic activity, with $\mathrm{CO}$ conversion of $97.2 \%$ at $250{ }^{\circ} \mathrm{C}$. Its catalytic stability is also outstanding during the temperature range of $250-400^{\circ} \mathrm{C}$. X-ray diffraction (XRD), $\mathrm{N}_{2}$ physisorption, and $\mathrm{H}_{2}$ temperature program reduction $\left(\mathrm{H}_{2}-\mathrm{TPR}\right)$ techniques were used to characterize the crystal phases, textures, and reduction properties of the $\mathrm{Cu} / \mathrm{Fe}_{2} \mathrm{O}_{3}$ catalysts. The results show that the generation of $\mathrm{CuFe}_{2} \mathrm{O}_{4}$, which has a spinel structure in stabilizing $\mathrm{Cu}$ microcrystals and is easier to be reduced at low temperature, resulting in enhancing their reduction properties and facilitating electrons transfer between $\mathrm{Cu}$ and $\mathrm{Fe}_{2} \mathrm{O}_{3}$, thus greatly improving the catalytic performance. Furthermore, $\left(\mathrm{NH}_{4}\right)_{2} \mathrm{CO}_{3}$ solution treatment of the as-prepared catalysts was performed to study the effect of bulk $\mathrm{CuO}$ existed in the $\mathrm{Cu} / \mathrm{Fe}_{2} \mathrm{O}_{3}$ catalysts. The result suggests that the bulk $\mathrm{CuO}$ is favor for $\mathrm{H}$ atom transfer between $\mathrm{Cu}$ and $\mathrm{Fe}_{2} \mathrm{O}_{3}$, thus promoting the reduction of $\mathrm{CuFe}_{2} \mathrm{O}_{4}$, finally improving the catalytic performance.
\end{abstract}

Key Words: Water gas shift; $\mathrm{Cu} / \mathrm{Fe}_{2} \mathrm{O}_{3}$ catalyst; Copper loading; $\mathrm{CuFe}_{2} \mathrm{O}_{4}$

Received: September 2, 2013; Revised: November 27, 2013; Published on Web: November 27, 2013.

"Corresponding author. Email: linxingyi@fzu.edu.cn; Tel: +86-591-83731234-8112.

The project was supported by the Education Department of Fujian Province Class A Science \& Technology Program, China (JA08021).

福建省教育厅 A 类科技项目(JA08021)

(c) Editorial office of Acta Physico-Chimica Sinica 


\section{1 引 言}

氢能以其清洁、高效、安全、可再生等特点, 被 视为 21 世纪最具发展潜力的能源. 氢燃料电池能 高效地将氢气的化学能转化为电能, 具有高效、节 能、环保的特点, 被认为是本世纪全新的环境友好 的利用氢能方式之一. 据报道, 目前大约 $95 \%$ 的 $\mathrm{H}_{2}$ 由原油、煤、天然气等经重整反应得到, 2,3 然而重整 气中含有的 $1 \%-10 \%$ (体积分数) CO 将导致燃料电 池铂电极中毒, 使燃料电池性能严重下降. ${ }^{4}$ 水煤气 变换(WGS) 反应不仅能够降低 CO 的浓度, 还可提 高重整气中 $\mathrm{H}_{2}$ 的含量, 从而引起燃料电池研究者的 广泛关注. ${ }^{5-7}$

近年来, 有关新型 WGS 催化剂的文献报道与 日俱增, 主要集中在负载型金属催化剂的研究上..$^{7-9}$ 其中, $\mathrm{Cu}$ 基催化剂因价格低廉, 且对 WGS 反应过程 发生的 $\mathrm{H}_{2} \mathrm{O}$ 解离吸附和 $\mathrm{CO}$ 氧化同时具有高活性, 促使研究者不遗余力地致力于以 $\mathrm{Cu}$ 为活性组分的 水煤气变换催化剂的开发. ${ }^{10-13} \mathrm{ZnO} 、 \mathrm{CeO}_{2} 、 \mathrm{ZrO}_{2}$ 、 $\mathrm{Al}_{2} \mathrm{O}_{3}$ 等金属氧化物常被选作载体, 研究发现, 载体 种类对 $\mathrm{Cu}$ 基 WGS 催化剂的性能影响显著, $\mathrm{Cu}$ 物种 和金属氧化物之间存在一定的相互作用, 改善了催 化剂的物理和化学性质, 表现出较 $\mathrm{Cu}$ 单质优异的催 化性能. ${ }^{14}$ 此外, $\mathrm{CuMoO}_{4}$ 和 $\mathrm{Cu}_{x} \mathrm{Ce}_{1-x} \mathrm{O}_{2-x}$ 等 $\mathrm{Cu}$ 基复合 氧化物也被证实具有较传统的 $\mathrm{Cu}-\mathrm{ZnO}$ 更优越的 WGS 反应性能. ${ }^{15,16}$ 近年来, 有关 $\mathrm{Cu}$ 或其氧化物作为 活性组分负载在具有还原性能的 $\mathrm{Fe}$ 载体上, 制得的 $\mathrm{Cu} / \mathrm{Fe}_{2} \mathrm{O}_{3}$ 双金属催化剂的报道日益增多..$^{17,18}$ 研究表 明, 所合成的催化剂通常含有大量的铁酸铜 $\left(\mathrm{CuFe}_{2} \mathrm{O}_{4}\right)$, 其在二甲醚重整、合成气制甲醇 ${ }^{19}$ 等领 域表现出良好的催化性能. Andreev 等 ${ }^{20,21}$ 还发现, 当 $\mathrm{Fe}$-系水煤气变换中少量 $\mathrm{Cu}$ 时, 由于形成了新 的 $\mathrm{Cu}$ 物种使其变换性能得到明显改善. Smirniotis 等 ${ }^{22,23}$ 发现 $\mathrm{Cu}$ 可进入 $\mathrm{Fe}_{3} \mathrm{O}_{4}$ 晶格, 从而改变催化剂的 物理化学性质.

Estrella 等 ${ }^{18}$ 运用原位 XRD 和原位 X 射线吸收 精细结构谱(XAFS) 考察了商品 $\mathrm{CuFe}_{2} \mathrm{O}_{4}$ (含有少量 的 $\mathrm{CuO}$ ) 的还原性能. 研究发现, 由于 $\mathrm{CuFe}_{2} \mathrm{O}_{4}$ 晶格中 存在 “metal $\leftrightarrow$ oxygen $\leftrightarrow$ metal” 结构, 增强了尖晶石结 构中 $\mathrm{Cu}^{2+}$ 和 $\mathrm{Fe}^{3+}$ 的稳定性, 使得 $\mathrm{CuFe}_{2} \mathrm{O}_{4}$ 较 $\mathrm{CuO}$ 和 $\mathrm{Fe}_{2} \mathrm{O}_{3}$ 更加难以还原, 在高于 $400{ }^{\circ} \mathrm{C}$ 时, 才能发生 $\mathrm{CuFe}_{2} \mathrm{O}_{4} \rightarrow \mathrm{Cu}+\mathrm{Fe}_{3} \mathrm{O}_{4}$ 或 $\mathrm{CuFe}_{5} \mathrm{O}_{8}$ 的还原. Estrella 等 ${ }^{18}$ 同时考察了其水煤气反应性能, 结合 XRD 数据发 现, 在 WGS 反应气氛中 $\mathrm{CuFe}_{2} \mathrm{O}_{4}$ 表面高度分散的
$\mathrm{CuO}$ 首先在 $175-250{ }^{\circ} \mathrm{C}$ 还原为单质 $\mathrm{Cu}$, 然后单质 $\mathrm{Cu}$ 进入 $\mathrm{CuFe}_{2} \mathrm{O}_{4}$ 晶格的八面体位中, 当温度高于 $250{ }^{\circ} \mathrm{C}$ 时, 单质 $\mathrm{Cu}$ 再从 $\mathrm{CuFe}_{2} \mathrm{O}_{4}$ 晶格的八面体位中 迁移出来, 出现 $\mathrm{Cu}$ 单质; 随着温度的升高, $\mathrm{CuFe}_{2} \mathrm{O}_{4}$ 复合氧化物中的 $\mathrm{CuO}$ 不稳定, 在高于 $250{ }^{\circ} \mathrm{C}$ 时, $\mathrm{Cu}^{2+}$ 离子会从 $\mathrm{CuFe}_{2} \mathrm{O}_{4}$ 晶格的八面体位中迁移出来, 发 生 $\mathrm{Cu}^{2+} \rightarrow \mathrm{Cu}$ 的还原, 从而使得催化剂表现出良好的 水煤气变换反应活性. 虽然 Estrella 等 ${ }^{18}$ 发现 $\mathrm{CuFe}_{2} \mathrm{O}_{4}$ 对 WGS 反应性能影响显著, 研究者认为 $\mathrm{Fe}_{2} \mathrm{O}_{3}$ 表面的非 $\mathrm{CuFe}_{2} \mathrm{O}_{4}$ 相 $\mathrm{Cu}$ 物种的物理化学性质 对 $\mathrm{Cu} / \mathrm{Fe}_{2} \mathrm{O}_{3}$ 催化剂也有较大的影响. ${ }^{23}$ 因此, 本文采 用共沉淀法制备了一系列不同 $\mathrm{Cu}$ 含量的 $\mathrm{Cu}-\mathrm{Fe}$ 水 煤气变换催化剂, 运用 $\mathrm{X}$ 射线衍射 $(\mathrm{XRD}) 、 \mathrm{~N}_{2}$ 物理吸 附和 $\mathrm{H}_{2}$ 程序升温还原 $\left(\mathrm{H}_{2}\right.$-TPR) 等表征手段研究其 结构及还原性能, 进而详细探讨 $\mathrm{Cu} / \mathrm{Fe}_{2} \mathrm{O}_{3}$ 催化剂表 面 $\mathrm{Cu}$ 物种和 $\mathrm{Fe}_{2} \mathrm{O}_{3}$ 之间的相互作用及其水煤气变换 性能之间的关系.

\section{2 实验部分}

\section{1 催化剂制备}

根据催化剂组成, 称取一定量的 $\mathrm{Cu}\left(\mathrm{NO}_{3}\right)_{2}$. $3 \mathrm{H}_{2} \mathrm{O}$ (分析纯) 和 $\mathrm{Fe}\left(\mathrm{NO}_{3}\right)_{3} \cdot 9 \mathrm{H}_{2} \mathrm{O}$ (分析纯) 溶解于 $400 \mathrm{~mL}$ 去离子水中; 按化学计量比称取一定量的 $\mathrm{KOH}$ (分析纯), 并配成 $500 \mathrm{~mL}$ 的溶液. 在摚拌条件 下, 采用并流共沉淀法, 将混合金属盐溶液和 $\mathrm{KOH}$ 溶液同时加入三口烧瓶中. 反应过程控制水浴温度 $80^{\circ} \mathrm{C}$, 反应体系 $\mathrm{pH}=10$; 待混合金属盐溶液滴加完 毕后, 在 $80^{\circ} \mathrm{C}$ 下, 继续摚拌并陈化 $4 \mathrm{~h}$. 所得沉淀物 经过离心、洗涤 8 次(脱除 $\mathrm{K}^{+}$离子等) $110^{\circ} \mathrm{C}$ 干燥 12 $\mathrm{h}$ 后, 在静态空气气氛中 $650^{\circ} \mathrm{C}$ 焙烧 $4 \mathrm{~h}$, 即得系列 $\mathrm{Cu} / \mathrm{Fe}_{2} \mathrm{O}_{3}$ 催化剂样品, 分别标记为 $\mathrm{CF}-X(X \%$ 为 $\mathrm{CuO}$ 的质量分数). 纯 $\mathrm{Fe}_{2} \mathrm{O}_{3}$ 的制备过程同上.

CF-20样品的还原预处理过程如下: 取适量共 沉淀法制备的 CF-20催化剂于管式炉中, 用 10\% (体 积分数) $\mathrm{H}_{2} / \mathrm{Ar}$ 混合气体分别升温还原至 $180 、 230$ 和 $300{ }^{\circ} \mathrm{C}$, 然后降至室温, 分别记作: CF20-180、CF20230 和 CF20-300.

CF-20 催化剂的碳酸铵处理流程为: 称取 $1.0 \mathrm{~g}$ 共沉淀法制备的 CF-20 催化剂, 将其浸泡在 $20 \mathrm{~mL}$ 、 $1 \mathrm{~mol} \cdot \mathrm{L}^{-1}$ 的碳酸铵 $\left.\left(\mathrm{NH}_{4}\right)_{2} \mathrm{CO}_{3}\right)$ 溶液中 $5 \mathrm{~h}$; 随后将未 溶解的固体样品过滤、洗涤直至 $\mathrm{pH}$ 为中性; 最后在 $120{ }^{\circ} \mathrm{C}$ 下干燥 $12 \mathrm{~h}$, 获得的样品记作 CF20-AC.

\section{2 催化剂表征}


XRD 分析采用荷兰 Panalytical 公司 $X^{\prime}$ per Pro 型粉末衍射仪. $X^{\prime}$ Celerator超能探测器, Co 靶 $(\lambda=$ $0.1789 \mathrm{~nm})$, 管电压 $40 \mathrm{kV}$, 管电流 $40 \mathrm{~mA}$, 扫描步长 为 $0.02^{\circ}$, 扫描速率 $0.25\left(^{\circ}\right) \cdot \mathrm{min}^{-1}$.

样品的比表面积和孔结构在美国 Micrometrics 公司 ASAP 2020 型气体吸附仪上测定. 准确称取一 定量样品, 在 $200^{\circ} \mathrm{C}$ 抽真空处理 $4 \mathrm{~h}$, 然后用 $\mathrm{N}_{2}$ 吸附 法在液氮温度 $\left(-196^{\circ} \mathrm{C}\right)$ 下测量. 样品的比表面积按 BET 方程计算, 孔体积和孔径分布则采用 BJH 法求 得.

$\mathrm{H}_{2}$ 程序升温还原实验在美国 Micrometrics 公司 AutoChem 2910 型自动化学吸附仪上进行. 在高纯 $\mathrm{He}\left(30 \mathrm{~mL} \cdot \mathrm{min}^{-1}\right)$ 气氛下, 以 $10^{\circ} \mathrm{C} \cdot \mathrm{min}^{-1}$ 速率升温 至 $300^{\circ} \mathrm{C}$, 保持 $30 \mathrm{~min}$ 后降至室温; 改用 $10 \% \mathrm{H}_{2} / \mathrm{Ar}$ 混合气 $\left(30 \mathrm{~mL} \cdot \mathrm{min}^{-1}\right)$ 吹扫样品, 待基线稳定以后, 以 $10^{\circ} \mathrm{C} \cdot \mathrm{min}^{-1}$ 速率升温至 $800^{\circ} \mathrm{C}$, 热导检测器(TCD) 记录耗 $\mathrm{H}_{2}$ 量.

\section{3 活性评价}

催化剂活性评价在固定床 $\mathrm{CO}$ 水煤气变换反应 活性测试装置上进行. 反应器为内径 $12 \mathrm{~mm}$ 的不锈 钢管, 采用程序升温控制反应温度. 活性测试条件 为: 常压、体积空速为 $4500 \mathrm{~h}^{-1}$ 、催化剂装填量 1.4 $\mathrm{mL}$ (20-40 目)、汽气比 1:1. 活性测试温度范围 200$400{ }^{\circ} \mathrm{C}$, 测试间隔 $50{ }^{\circ} \mathrm{C}$, 每个温度点保持 $4 \mathrm{~h}$. 原料 气组成(体积分数): $10 \% \mathrm{CO}, 60 \% \mathrm{H}_{2}, 12 \% \mathrm{CO}_{2}$, 其余 为 $\mathrm{N}_{2}$, 反应前后 $\mathrm{CO}$ 含量通过福立 GC9750 型气相色 谱仪检测. 催化剂活性用 $\mathrm{CO}$ 转化率 $\left(\eta_{\mathrm{co}}\right)$ 表示:

$$
\eta_{\mathrm{CO}}=\left(1-\varphi_{\mathrm{CO}} / \varphi_{\mathrm{CO}}\right) /\left(1+\varphi_{\mathrm{CO}}\right) \times 100 \%
$$

式中 $\varphi_{\mathrm{CO}}$ 为原料气中 $\mathrm{CO}$ 的体积分数, $\varphi_{\mathrm{CO}}$ 为变换气出 $\square \mathrm{CO}$ 的体积分数.

稳定性评价: 在完成第一轮活性测试之后, 继 续将其置于 $400{ }^{\circ} \mathrm{C}$ 下保持 $10 \mathrm{~h}$, 再降温到 $200^{\circ} \mathrm{C}$ 并 稳定 $2 \mathrm{~h}$, 重新在 $200-400{ }^{\circ} \mathrm{C}$ 范围内进行第二轮水 煤气变换反应性能测试.

\section{3 结果与讨论}

\section{1 铜负载量对 $\mathrm{Cu} / \mathrm{Fe}_{2} \mathrm{O}_{3}$ 催化剂 WGS 性能的 影响}

不同 $\mathrm{CuO}$ 负载量的 $\mathrm{Cu} / \mathrm{Fe}_{2} \mathrm{O}_{3}$ 催化剂 WGS 反应 性能评价结果如图 1 所示. 从图中可以看出, 纯 $\mathrm{Fe}_{2} \mathrm{O}_{3}$ 在 $200-300{ }^{\circ} \mathrm{C}$ 温区内 $\mathrm{CO}$ 转化率几乎为零, $400{ }^{\circ} \mathrm{C}$ 时也仅有 $60.7 \%$ 的转化率; $\mathrm{CuO}$ 的引入显著 提高了催化剂的 $\mathrm{WGS}$ 变换反应活性, 随着 $\mathrm{CuO}$ 负

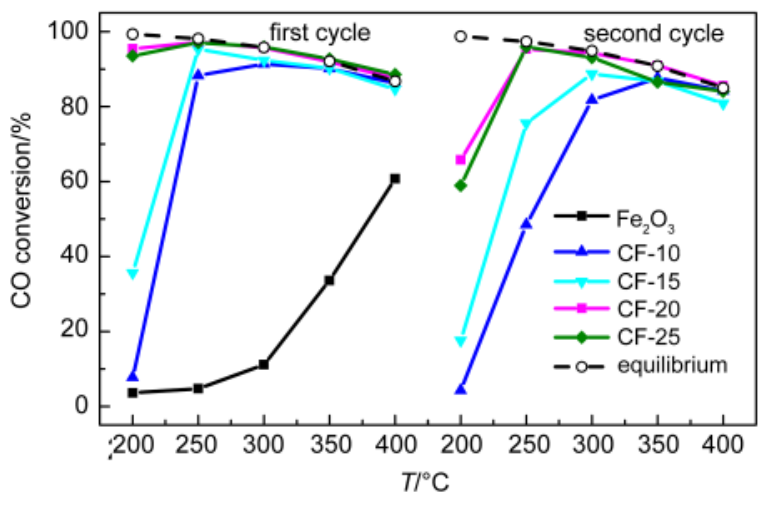

图 1 不同 $\mathrm{CuO}$ 负载量 $\mathrm{Cu} / \mathrm{Fe}_{2} \mathrm{O}_{3}$ 催化剂 水煤气变换(WGS)活性图

Fig.1 Catalytic activity of $\mathrm{Cu} / \mathrm{Fe}_{2} \mathrm{O}_{3}$ catalysts with different $\mathrm{CuO}$ loadings for the water gas shift (WGS) reaction

CF-10 is the label of $\mathrm{Cu} / \mathrm{Fe}_{2} \mathrm{O}_{3}$ catalyst with $10 \%(w) \mathrm{CuO}$ loading, and the CF-15, Cf-20, and CF-25 are for $15 \%, 20 \%$, and $25 \%(w)$ $\mathrm{CuO}$ loadings, respectively.

载量的增加, 催化剂在 $200{ }^{\circ} \mathrm{C}$ 的 $\mathrm{CO}$ 转化率提高显 著, 当 $\mathrm{CuO}$ 负载量为 $10 \%(w$, 质量分数, CF-10) 时, 催化剂还未起活, 但当 $\mathrm{CuO}$ 负载量达 $20 \%(w)$ 时 (CF-20), $\mathrm{CO}$ 的转化率高于 $90 \%$; 进一步提高 $\mathrm{CuO}$ 的 负载量 $(>20 \%(w))$, 催化剂活性基本保持不变. 由此 可见, $\mathrm{Cu} / \mathrm{Fe}_{2} \mathrm{O}_{3}$ 催化剂 WGS 活性温区随 $\mathrm{CuO}$ 负载量 的增多而向低温方向移动, 说明该催化剂的低温活 性组分可能为 $\mathrm{Cu}$ 物种; 同时, $\mathrm{Cu}$ 作为助剂提高了 $\mathrm{Fe}$ 系催化剂的高温 WGS 反应活性. ${ }^{24}$ 耐热后(图 1, second cycle), $\mathrm{CuO}$ 负载量高于 $20 \%(w)$ 的样品也表现 出良好的热稳定性, 其中 CF- 20 样品仅在 $200{ }^{\circ} \mathrm{C}$ 时 $\mathrm{CO}$ 转化率有所下降, 而在 $250-400{ }^{\circ} \mathrm{C}$ 温度区间内, 活性基本保持不变. 为了进一步考察 CF-20样品的 稳定性, 将该样品在 $400{ }^{\circ} \mathrm{C}$ 下进行 WGS 反应活性 评价, 结果如图 2 所示. 从图中可以看出, 经过 $46 \mathrm{~h}$ 的活性测试, $\mathrm{CO}$ 转化率未见明显下降, 接近该温度 下的平衡转化率, 说明该样品的耐热稳定性和使用 寿命均较优异. 由此可以看出, $\mathrm{Cu} / \mathrm{Fe}_{2} \mathrm{O}_{3}$ 催化剂不是 $\mathrm{CuO}$ 和 $\mathrm{Fe}_{2} \mathrm{O}_{3}$ 的简单线性叠加, $\mathrm{CuO}$ 的引入改变了 $\mathrm{Cu} / \mathrm{Fe}_{2} \mathrm{O}_{3}$ 催化剂的组成、结构等, 从而影响催化剂的 活性物种的种类、活性中心的起活温度等.

\subsection{XRD 表征}

图 3 为系列新鲜制备 $\mathrm{Cu} / \mathrm{Fe}_{2} \mathrm{O}_{3}$ 催化剂的 $\mathrm{XRD}$ 谱 图, “\#”标记为单斜相 CuO(JCPDS: 05-0661)的特征 衍射峰、“*”为斜方相 $\mathrm{Fe}_{2} \mathrm{O}_{3}$ (JCPDS: 89-0596)的特征 衍射峰、“†”为四方相 $\mathrm{CuFe}_{2} \mathrm{O}_{4}$ 复合氧化物(JCPDS: 34-0425)的特征衍射峰. 由图 3 可以看出, 谱图中各 


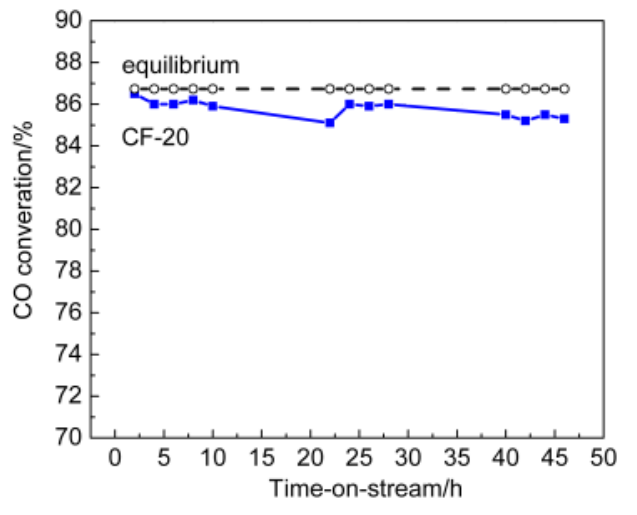

图 2 CF-20样品在 $400{ }^{\circ} \mathrm{C}$ 下的 WGS 活性图

Fig.2 Catalytic activity of CF-20 catalyst for the WGS reaction at $400{ }^{\circ} \mathrm{C}$

组分的特征衍射峰强度随 $\mathrm{CuO}$ 负载量的不同而有 所差异: 随着 $\mathrm{CuO}$ 负载量的增加, $\mathrm{Fe}_{2} \mathrm{O}_{3}$ 衍射峰呈现 出强度减小且峰宽变大的趋势, 这可能是由于一方 面样品中 $\mathrm{Fe}_{2} \mathrm{O}_{3}$ 含量减少, 另一方面负载 $\mathrm{CuO}$ 和 $\mathrm{CuFe}_{2} \mathrm{O}_{4}$ 物种起着分散阻隔作用, 抑制了 $\mathrm{Fe}_{2} \mathrm{O}_{3}$ 晶粒 生长; 当 $\mathrm{CuO}$ 添加量为 $15 \%(w, \mathrm{CF}-15)$ 时, 出现了微 弱的 $\mathrm{CuFe}_{2} \mathrm{O}_{4}$ 特征衍射峰, 继续增大 $\mathrm{CuO}$ 负载量, $\mathrm{CuFe}_{2} \mathrm{O}_{4}$ 的衍射峰也随之增强; 特别值得注意的是, 除 CF-20样品外, 其余各个样品均在 $2 \theta=45.3^{\circ}$ 出现 了 $\mathrm{CuO}$ 衍射峰. 仔细观察各峰强度的变化可以发 现: $\mathrm{CuO}$ 含量从 $15 \%(w)$ 提高到 $20 \%(w)$ 时, $\mathrm{CuFe}_{2} \mathrm{O}_{4}$ 的含量显著增大; 但从 $20 \%(w)$ 提高到 $25 \%(w)$, $\mathrm{CuFe}_{2} \mathrm{O}_{4}$ 的衍射峰强度变化不显著, 说明 $\mathrm{CuFe}_{2} \mathrm{O}_{4}$ 的 形成与 $\mathrm{Cu}: \mathrm{Fe}$ 原子比密切相关, 当 $\mathrm{Cu}: \mathrm{Fe}$ 原子比为 1 : 2 (CF-20) 时, 形成了大量的 $\mathrm{CuFe}_{2} \mathrm{O}_{4}$, 同时 $\mathrm{CuFe}_{2} \mathrm{O}_{4}$ 的生成有利于 $\mathrm{CuO}$ 的分散, 从而观察不到 $\mathrm{CuO}$ 的衍 射峰; 随着 $\mathrm{CuO}$ 负载量的进一步增大将发生 $\mathrm{CuO}$ 晶 粒团聚, 出现体相的 $\mathrm{CuO}$ 衍射峰.

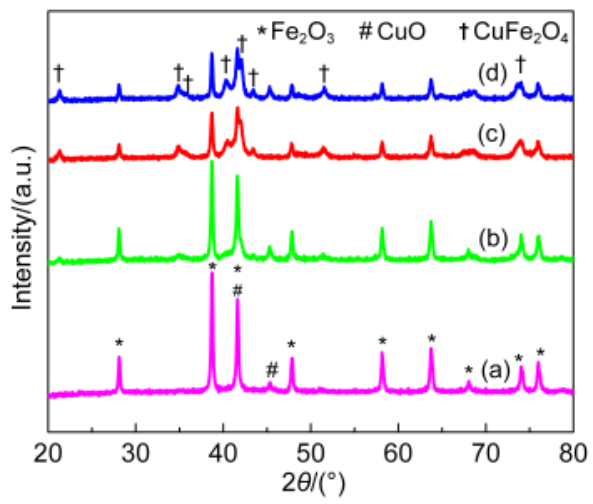

图 3 不同 $\mathrm{CuO}$ 负载量 $\mathrm{Cu} / \mathrm{Fe}_{2} \mathrm{O}_{3}$ 催化剂的 $\mathrm{XRD}$ 图谱

Fig.3 XRD patterns of $\mathrm{Cu} / \mathrm{Fe}_{2} \mathrm{O}_{3}$ catalysts with different $\mathrm{CuO}$ loadings

(a) CF-10; (b) CF-15; (c) CF-20; (d) CF-25
关联活性数据可以发现: CF-10样品未观察到 $\mathrm{CuFe}_{2} \mathrm{O}_{4}$ 物种, 其 WGS 反应活性和稳定性也最差; CF-20 样品相较于 CF-15 样品, 催化剂中 $\mathrm{CuFe}_{2} \mathrm{O}_{4}$ 的 含量显著增大, 同时催化剂的低温 $\left(200-250{ }^{\circ} \mathrm{C}\right)$ WGS 反应活性大幅提高, 耐热稳定性也明显增强; 当 $\mathrm{CuO}$ 负载量 $\geqslant 20 \%(w)$ 时, 催化剂中 $\mathrm{CuFe}_{2} \mathrm{O}_{4}$ 的含 量基本保持不变, 催化剂WGS 反应活性和稳定性与 CF-20 样品基本相当. 由此可见, $\mathrm{CuFe}_{2} \mathrm{O}_{4}$ 物种的出 现及其含量的变化对 $\mathrm{Cu} / \mathrm{Fe}_{2} \mathrm{O}_{3}$ 催化剂的 WGS 反应 性能影响显著.

\section{3 织构表征}

运用 $\mathrm{N}_{2}$ 物理吸附实验测定催化剂的织构特征, 表征结果见表 1 .

从表中可以看出, 负载 $\mathrm{CuO}$ 后各样品的织构参 数均发生了一定程度的变化. 结合 XRD 表征结果可 知: CF-10 样品由于出现体相的 $\mathrm{CuO}$, 从而使部分 $\mathrm{Fe}_{2} \mathrm{O}_{3}$ 孔道被覆盖, 孔容减小, 平均孔径增大; 当 $\mathrm{CuO}$ 的负载量大于 $15 \%(w)$ 时, 由于生成新的 $\mathrm{CuFe}_{2} \mathrm{O}_{4}$ 物 种, 导致 $\mathrm{Fe}_{2} \mathrm{O}_{3}$ 骨架发生重构, 织构特征随之改变. 其 中 CF-20样品的 BET 比表面积、孔容和孔径均最大, 这可能是由于 $\mathrm{CuO}$ 呈高分散状态; 当进一步增加 $\mathrm{CuO}$, 孔容和孔径均减小, 说明多余的 $\mathrm{CuO}$ 可能分 布于载体 $\mathrm{Fe}_{2} \mathrm{O}_{3}$ 的孔道内壁. 比较活性与孔结构数据 可知, 在所研究的铜含量范围内, 当 $\mathrm{CuO}$ 负载量低 于 $20 \%(w)$ 时, $\mathrm{Cu} / \mathrm{Fe}_{2} \mathrm{O}_{3}$ 催化剂的低温活性和孔容、 比表面积存在着线性对应关系; 当 $\mathrm{CuO}$ 负载量高于 $20 \%(w)$ 时, 虽然催化剂的比表面积和孔容变小, 但 是催化剂的活性基本保持不变, 说明 $\mathrm{Cu} / \mathrm{Fe}_{2} \mathrm{O}_{3}$ 催化 剂的织构特征不是影响催化剂 WGS 反应性能的唯 一因素.

\section{4 还原性能表征}

图 4 是 $\mathrm{Cu} / \mathrm{Fe}_{2} \mathrm{O}_{3}$ 系列催化剂在 $50-800{ }^{\circ} \mathrm{C}$ 范围 内进行 $\mathrm{H}_{2}$-TPR 表征的结果. 纯 $\mathrm{Fe}_{2} \mathrm{O}_{3}$ 在 $450{ }^{\circ} \mathrm{C}$ 处的 还原峰对应于 $\mathrm{Fe}_{2} \mathrm{O}_{3} \rightarrow \mathrm{Fe}_{3} \mathrm{O}_{4}$ 的还原, $500-800{ }^{\circ} \mathrm{C}$ 的

表 1 不同 $\mathrm{CuO}$ 负载量的 $\mathrm{Cu} / \mathrm{Fe}_{2} \mathrm{O}_{3}$ 织构参数

Table 1 Texture parameters of the $\mathrm{Cu} / \mathrm{Fe}_{2} \mathrm{O}_{3}$ catalysts with different $\mathrm{CuO}$ contents

\begin{tabular}{|c|c|c|c|}
\hline Sample & $\frac{S_{\mathrm{BET}}}{\left(\mathrm{m}^{2} \cdot \mathrm{g}^{-1}\right)}$ & $\frac{\text { Pore volume }}{\left(\mathrm{cm}^{3} \cdot \mathrm{g}^{-1}\right)}$ & $\frac{\text { Average pore size }}{\mathrm{nm}}$ \\
\hline $\mathrm{Fe}_{2} \mathrm{O}_{3}$ & 26.0 & 0.34 & 58.9 \\
\hline CF-10 & 11.1 & 0.18 & 81.3 \\
\hline CF-15 & 13.9 & 0.22 & 72.9 \\
\hline CF-20 & 16.1 & 0.37 & 92.4 \\
\hline CF-25 & 15.6 & 0.24 & 68.7 \\
\hline
\end{tabular}




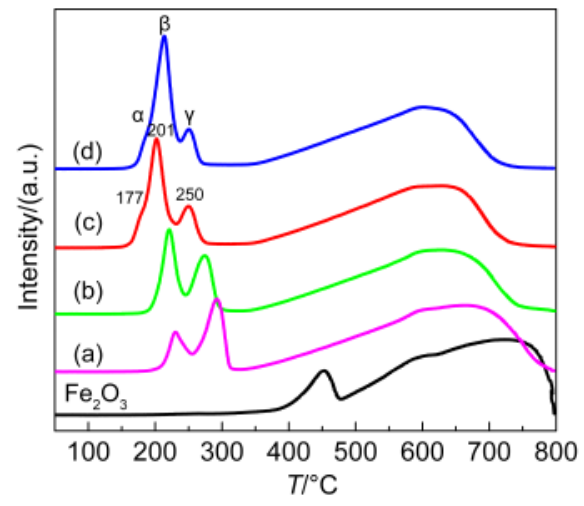

图 4 不同 $\mathrm{CuO}$ 负载量 $\mathrm{Cu} / \mathrm{Fe}_{2} \mathrm{O}_{3}$ 催化剂的 TPR 图谱

Fig.4 TPR profiles of $\mathrm{Cu} / \mathrm{Fe}_{2} \mathrm{O}_{3}$ catalysts with different $\mathrm{CuO}$ loadings

(a) CF-10; (b) CF-15; (c) CF-20; (d) CF-25

宽还原峰对应于 $\mathrm{Fe}_{3} \mathrm{O}_{4} \rightarrow \mathrm{FeO} \rightarrow \mathrm{Fe}$ 的还原..$^{20,25}$ 从图中 不难看出, $\mathrm{Cu}$ 的引入使得 $\mathrm{Fe}_{2} \mathrm{O}_{3}$ 在 $450{ }^{\circ} \mathrm{C}$ 处的还原 峰消失, 在 $150-300{ }^{\circ} \mathrm{C}$ 温度范围内出现新的还原 峰: $\mathrm{CuO}$ 负载量 $\leqslant 20 \%(w)$ 的三个样品, 随着 $\mathrm{CuO}$ 负 载量的增加, 整个还原峰向低温方向移动, 且随着 $\mathrm{CuO}$ 负载量的增大, 该区间的还原峰逐渐由两个分 裂为三个; 继续增加 $\mathrm{CuO}$ 负载量, $\mathrm{CF}-25$ 样品相对于 CF-20样品, 整体还原峰又向高温方向移动.

关于 $\mathrm{Cu} / \mathrm{Fe}_{2} \mathrm{O}_{3}$ 催化剂还原峰归属的报道较少, 但有关 $\mathrm{CuFe}_{2} \mathrm{O}_{4}$ 氧化还原性能的研究较多. Yang 等 ${ }^{26}$ 发现, $\mathrm{CuFe}_{2} \mathrm{O}_{4}$ 的 $\mathrm{H}_{2}$-TPR 曲线包含两个峰: 分别位于 $187^{\circ} \mathrm{C}$ 尖峰和 $600{ }^{\circ} \mathrm{C}$ 的宽峰, 归属为 $\mathrm{CuFe}_{2} \mathrm{O}_{4}$ 还原 为 $\mathrm{Cu}$ 和 $\mathrm{Fe}_{3} \mathrm{O}_{4}$ 以及 $\mathrm{Fe}_{3} \mathrm{O}_{4}$ 还原为 $\mathrm{Fe}$. Kameoka ${ }^{27}$ 和 Faungnawakij ${ }^{28}$ 等也观察到类似的还原峰, 并将峰温 位于 $200{ }^{\circ} \mathrm{C}$ 以下的还原峰归属为 $\mathrm{CuFe}_{2} \mathrm{O}_{4}$ 的还原. 但是, 图 4 中在 $150-300{ }^{\circ} \mathrm{C}$ 温度范围内, 各 $\mathrm{Cu} / \mathrm{Fe}_{2} \mathrm{O}_{3}$ 样品均观察到多个(两个或三个)还原峰的叠加, 为 此我们对催化剂进行进一步的还原预处理, 进而探 究其还原峰的归属.

经不同温度还原预处理的 CF-20样品的 XRD 表征结果如图 5(a)所示. 经 $180{ }^{\circ} \mathrm{C}$ 还原预处理后, $2 \theta=21.3^{\circ}, 40.5^{\circ}$ 处 (标记为 “十”) 的 $\mathrm{CuFe}_{2} \mathrm{O}_{4}$ 特征衍射 峰明显变弱, 且在 $2 \theta=50.6^{\circ}$ 处(标记为 “\#”)出现微弱 的 $\mathrm{Cu}$ 单质的衍射峰, 说明部分 $\mathrm{CuFe}_{2} \mathrm{O}_{4}$ 在 $180{ }^{\circ} \mathrm{C}$ 下 即发生还原; 然而 $\mathrm{Fe}_{2} \mathrm{O}_{3}$ 的特征衍射峰(标记为“*”) 较 CF-20 样品未发生明显的变化, 结合图 5(b) 的 $\mathrm{TPR}$ 曲线可知, $\gamma$ 峰应归属为 $\mathrm{Fe}_{2} \mathrm{O}_{3}$ 的还原. 当还原温 度升至 $230{ }^{\circ} \mathrm{C}$ 时 (记为 $\mathrm{CF}-20-230$ ), 产物中 $\mathrm{Fe}_{3} \mathrm{O}_{4}$ (标 记为 “†”)和单质 $\mathrm{Cu}$ 的特征衍射峰明显增强, 说明该 温度下 $\mathrm{Fe}_{2} \mathrm{O}_{3}$ 开始被还原, 这与图 5(b)中 CF-20 的 $\gamma$ 峰
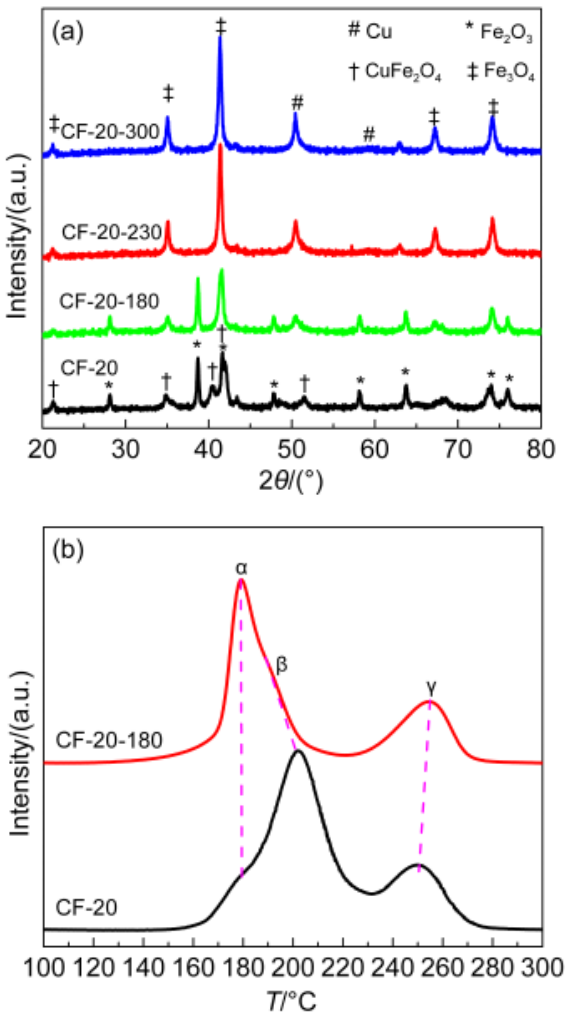

图 5 经不同温度还原预处理 CF-20 催化剂的 XRD

图谱(a)和 $\mathrm{H}_{2}-\mathrm{TPR}$ 曲线(b)

Fig.5 XRD patterns (a) and $\mathrm{H}_{2}$-TPR profiles (b) of CF-20 catalysts after reduction treatment at different temperatures

CF-20-180, CF-20-230, and CF-20-300 denote the CF-20 sample treated at 180,230 , and $300{ }^{\circ} \mathrm{C}$, respectively.

的起始还原峰温相一致; 进一步将还原温度升至 $300{ }^{\circ} \mathrm{C}, \mathrm{Fe}_{3} \mathrm{O}_{4}$ 的特征衍射峰继续增强, 进一步证实 了 $\gamma$ 峰的归属. 从图 5(b)中我们还可以看出, CF-20180 样品的 $\alpha$ 和 $\beta$ 还原峰面积较 CF-20样品发生了明 显的变化, 这是由于经 $180^{\circ} \mathrm{C}$ 还原后, 还原产物中 仍保留了少量的 $\mathrm{CuFe}_{2} \mathrm{O}_{4}$, 同时还原产物中单质 $\mathrm{Cu}$ 较易发生氧化转变为 $\mathrm{CuO}$, 从而表现为 $\mathrm{CF}-20-180$ 的 $\mathrm{CuO}$ 还原峰变大, 由此, $\alpha$ 峰可归属为 $\mathrm{CuO}$ 的还 原, 而 $\beta$ 为催化剂中 $\mathrm{CuFe}_{2} \mathrm{O}_{4}$ 的还原.

综合上述表征结果可以发现, 由于 $\mathrm{CuO}$ 的引 入, 极大地促进了 $\mathrm{Fe}_{2} \mathrm{O}_{3} \rightarrow \mathrm{Fe}_{3} \mathrm{O}_{4}$ 的还原(还原峰温降 幅大于 $\left.150{ }^{\circ} \mathrm{C}\right)$, 这可能是由于 $\mathrm{CuO}$ 较易还原, 其还 原后可以吸附氢; 同时, 催化剂各组分间存在较强 的相互作用, 使 $\mathrm{Cu}$ 可以向较难还原的 $\mathrm{Fe}$ 溢流原子 氢, 促使 $\mathrm{Fe}$ 在较低温度下向低价态转化 ${ }^{26}$ 当 $\mathrm{CuO}$ 负 载量较低时, 仅观察到 $\alpha$ 和 $\gamma$ 两个还原峰, 当 $\mathrm{CuO}$ 负 载量达到 $20 \%(w)$ 以上时, 还原峰分裂为三个, 这与 $\mathrm{XRD}$ 的物相表征结果相一致; 特别值得关注的是, 
$\mathrm{CuO}$ 负载量的变化对还原峰温的影响较大, $\mathrm{CuFe}_{2} \mathrm{O}_{4}$ 物种的出现使还原峰温进一步降低, 其中 CF-20样 品的还原峰温最低; 此外, 虽然 CF-25 样品较 CF-20 样品, $\alpha$ 和 $\gamma$ 峰面积略有减小, $\beta$ 峰面积略微增大, 但是 其整体还原峰却向高温方向移动, 说明 $\mathrm{CuFe}_{2} \mathrm{O}_{4}$ 物 种不是影响催化剂还原性能的唯一因素, 催化剂表 面 $\mathrm{CuO}$ 物种也起着至关重要的作用.

\section{$3.5\left(\mathrm{NH}_{4}\right)_{2} \mathrm{CO}_{3}$ 溶液处理}

据报道, ${ }^{29,30} \mathrm{CuO} / \mathrm{Al}_{2} \mathrm{O}_{3}$ 催化剂经 $1 \mathrm{~mol} \cdot \mathrm{L}^{-1}$ 的 $\left(\mathrm{NH}_{4}\right)_{2} \mathrm{CO}_{3}$ 溶液处理可以在不移除高度分散的 $\mathrm{CuO}$ 和 $\mathrm{CuAl}_{2} \mathrm{O}_{4}$ 尖晶石的前提下, 仅除去不与载体发 生相互作用的孤立的 $\mathrm{CuO}$. 通过使用 $1 \mathrm{~mol} \cdot \mathrm{L}^{-1}$ 的 $\left(\mathrm{NH}_{4}\right)_{2} \mathrm{CO}_{3}$ 溶液处理合成的 CF-20样品发现, 在处理 过程中, $\left(\mathrm{NH}_{4}\right)_{2} \mathrm{CO}_{3}$ 溶液的颜色由无色逐渐变为蓝 色, 说明发生了 $\mathrm{CuO}$ 脱除过程, 形成 $\mathrm{Cu}^{2+}$ 溶液; 从图 6(a)中也可以看出, 经 $\left(\mathrm{NH}_{4}\right)_{2} \mathrm{CO}_{3}$ 溶液处理后的 $\mathrm{CF}-$ 20-AC样品的 $\mathrm{Fe}_{2} \mathrm{O}_{3}$ 衍射峰强度变强, 表明随 $\mathrm{CuO}$ 的 移除, 其相对强度有所增大.

经 $\left(\mathrm{NH}_{4}\right)_{2} \mathrm{CO}_{3}$ 溶液处理前后的 CF-20 样品的 $\mathrm{H}_{2}-$
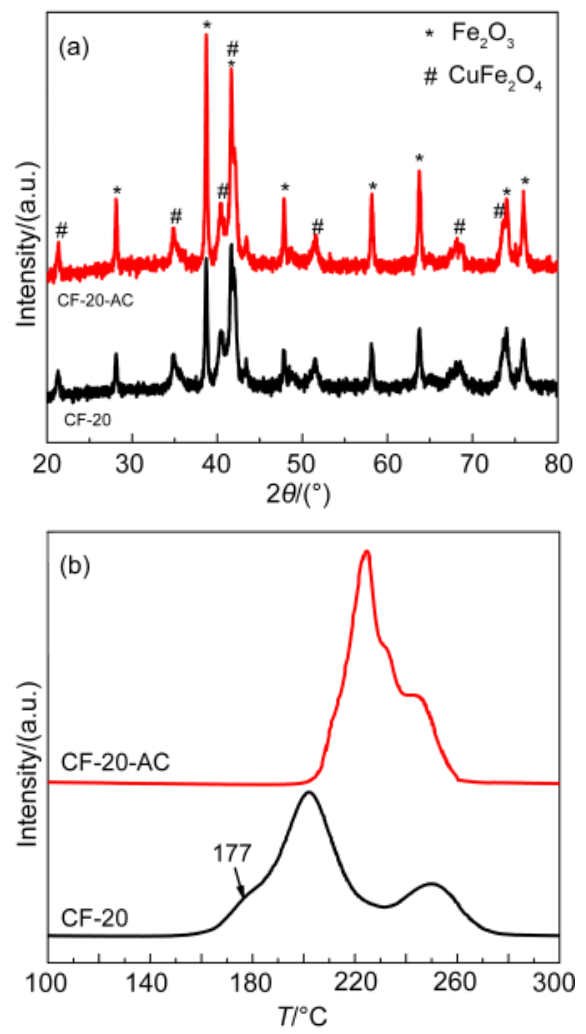

图 $6 \mathrm{CF}-20$ 催化剂经 $\left(\mathrm{NH}_{4}\right)_{2} \mathrm{CO}_{3}$ 溶液处理前后的 XRD 图谱(a)和 $\mathrm{H}_{2}-\mathrm{TPR}$ 曲线(b)

Fig.6 XRD patterns (a) and $\mathrm{H}_{2}$-TPR profiles (b) of CF-20 sample before and after treated with $\left(\mathrm{NH}_{4}\right)_{2} \mathrm{CO}_{3}$ solution CF-20-AC: after treating the CF-20 sample with $\left(\mathrm{NH}_{4}\right)_{2} \mathrm{CO}_{3}$ solution
表 2 CF-20 和 CF-20-AC 样品的 WGS 反应活性数据

Table 2 Catalytic activities of the WGS reaction for CF-20 and CF-20-AC samples

\begin{tabular}{cccccc}
\hline \multirow{2}{*}{ Sample } & \multicolumn{5}{c}{ CO conversion/\% } \\
\cline { 2 - 6 } & $200{ }^{\circ} \mathrm{C}$ & $250{ }^{\circ} \mathrm{C}$ & $300{ }^{\circ} \mathrm{C}$ & $350{ }^{\circ} \mathrm{C}$ & $400{ }^{\circ} \mathrm{C}$ \\
\hline CF-20 & 95.4 & 97.2 & 95.7 & 91.9 & 87.4 \\
Cf-20-AC & 89.5 & 96.7 & 93.7 & 90.5 & 85.2 \\
\hline
\end{tabular}

TPR 如图 6(b)所示, 处理后 CF-20样品位于 $177^{\circ} \mathrm{C}$ 归属为 $\mathrm{CuO}$ 的还原峰消失, 同时 $\mathrm{CuFe}_{2} \mathrm{O}_{4}$ 的还原峰 也向高温方向移动了约 $30{ }^{\circ} \mathrm{C}$. 这说明 $\mathrm{CuO}$ 和 $\mathrm{CuFe}_{2} \mathrm{O}_{4}$ 之间存在着较强的相互作用, 适量的 $\mathrm{CuO}$ 存在有利于 $\mathrm{H}$ 原子的传递, 进而促进 $\mathrm{CuFe}_{2} \mathrm{O}_{4}$ 的还 原, 这与 CF-25 样品较 CF-20样品向还原峰高温方 向移动的现象相一致. 表 2 中列出了处理前后的 CF20 样品的 WGS 反应活性数据, 相较于 CF-20, CF20-AC 样品的 $\mathrm{CO}$ 转化率在 $200-400{ }^{\circ} \mathrm{C}$ 测试温区内 略微的降低, 其中 $200^{\circ} \mathrm{C}$ 温度点的降幅最大, 大约 为 $5.9 \%$. 这说明 $\mathrm{CF}-20$ 催化剂中经 $\left(\mathrm{NH}_{4}\right)_{2} \mathrm{CO}_{3}$ 溶液处 理后, 移除的 $\mathrm{CuO}$ 颗粒对催化剂的水煤气变换性能 有一定的影响, 结合图 1 的活性数据可以发现, $\mathrm{CuFe}_{2} \mathrm{O}_{4}$ 物种的出现有利于大幅提高 $\mathrm{Cu} / \mathrm{Fe}_{2} \mathrm{O}_{3}$ 催化 剂的低温 WGS 变换反应活性; 同时 $\mathrm{CuFe}_{2} \mathrm{O}_{4}$ 特殊的 尖晶石结构有利于提升 $\mathrm{Cu}$ 微晶的热稳定性, 从而改 善催化剂的耐热稳定性; 但是 $\mathrm{CuFe}_{2} \mathrm{O}_{4}$ 物种不是影 响 $\mathrm{Cu} / \mathrm{Fe}_{2} \mathrm{O}_{3}$ 催化剂性能的唯一因素, 催化剂中存在 的适量 $\mathrm{CuO}$ 可促进 $\mathrm{CuFe}_{2} \mathrm{O}_{4}$ 物种的还原, 进而提高 催化剂的WGS 性能.

\section{4 结 论}

采用共沉淀法成功制备了系列 WGS 反应性能 优异的 $\mathrm{Cu} / \mathrm{Fe}_{2} \mathrm{O}_{3}$ 催化剂, 当 $\mathrm{CuO}$ 的负载量为 $20 \%$ $(w)$ 时, 在整个测试温区范围内 $\left(200-400{ }^{\circ} \mathrm{C}\right)$ 活性和 热稳定性最优. 研究发现, 铜主要以 $\mathrm{CuFe}_{2} \mathrm{O}_{4}$ 复合金 属氧化物和 $\mathrm{CuO}$ 形式存在; $\mathrm{CuFe}_{2} \mathrm{O}_{4}$ 物种的产生, 改 变了催化剂的织构特征, 并使其还原性能得到改 善; 同时独立相 $\mathrm{CuO}$ 的存在, 有利于 $\mathrm{H}$ 原子在各组 分传递，从而促进催化剂的 $\mathrm{CuFe}_{2} \mathrm{O}_{4}$ 的还原; $\mathrm{CuFe}_{2} \mathrm{O}_{4}$ 物种的生成和适量 $\mathrm{CuO}$ 的存在极大改善了 $\mathrm{Cu} / \mathrm{Fe}_{2} \mathrm{O}_{3}$ 催化剂的 WGS 反应性能.

\section{References}

(1) Hoffmann, P. Tomorrow's Energy: Hydrogen, Fuel Cells, and the Prospects for a Cleaner Planet; MIT Press: Cambridge, Massachusetts, 2001. 
(2) Spivey, J. J. Catal. Today 2005, 100, 171. doi: 10.1016/j. cattod.2004.12.011

(3) Tonkovich, A. Y.; Zilka, J. L.; LaMont, M. J.; Wang, Y.; Wegeng, R. S. Chem. Eng. Sci. 1999, 54, 2947. doi: 10.1016/ S0009-2509(98)00346-7

(4) Suh, D. J.; Kwak, C.; Kim, J. H.; Kwon, S. M.; Park, T. J. J. Power Sources 2005, 142, 70. doi: 10.1016/j.jpowsour. 2004.09.012

(5) Andreeva, D.; Idakiev, V.; Tabakova, T.; Andreev, A. J. Catal. 1996, 158, 354. doi: 10.1006/jcat.1996.0035

(6) Li, Y.; Fu, Q.; Flytzani-Stephanopoulos, M. Appl. Catal. B: Environ. 2000, 27, 179. doi: 10.1016/S0926-3373(00)00147-8

(7) Zhai, Y.; Pierre, D.; Si, R.; Deng, W.; Ferrin, P.; Nilekar, A. U.; Peng, G.; Herron, J. A.; Bell, D. C.; Saltsburg, H.; Mavrikakis, M.; Flytzani-Stephanopoulos, M. Science 2010, 329, 1633. doi: 10.1126/science. 1192449

(8) Zhang, Y.; Zhan, Y.; Chen, C.; Cao, Y.; Lin, X.; Zheng, Q. Int. J. Hydrog. Energy 2012, 37, 12292. doi: 10.1016/j.jhydene. 2012.06 .025

(9) She, Y.; Zheng, Q.; Li, L.; Zhan, Y.; Chen, C.; Zheng, Y.; Lin, X. Int. J. Hydrog. Energy 2009, 34, 8929. doi: 10.1016/j.ijhydene. 2009.08.062

(10) Sagata, K.; Imazu, N.; Yahiro, H. Catal. Today 2013, 201, 145. doi: 10.1016/j.cattod.2012.03.064

(11) Kubacka, A.; Si, R.; Michorczyk, P.; Martínez-Arias, A.; Xu, W.; Hanson, J. C.; Rodriguez, J. A.; Fernández-García, M. Appl. Catal. B: Environ. 2013, 132-133, 423.

(12) Sagata, K.; Yahiro, H. B. Chem. Soc. Jpn. 2012, 85, 511. doi: 10.1246/bcsj.20110283

(13) Rasmussen, D. B.; Janssens, T. V. W.; Temel, B.; Bligaard, T.; Hinnemann, B.; Helveg, S.; Sehested, J. J. Catal. 2012, 293, 205. doi: 10.1016/j.jcat.2012.07.001

(14) Li, L.; Song, L.; Wang, H.; Chen, C.; She, Y.; Zhan, Y.; Lin, X.; Zheng, Q. Int. J. Hydrog. Energy 2011, 36, 8839. doi: 10.1016/j. ijhydene.2011.04.137

(15) Wang, X.; Rodriguez, J. A.; Hanson, J. C.; Gamarra, D.; Martínez-Arias, A.; Fernández-García, M. J. Phys. Chem. B 2005, 110,428
(16) Wen, W.; Jing, L.; White, M.; Marinkovic, N.; Hanson, J.; Rodriguez, J. Catal. Lett. 2007, 113, 1. doi: 10.1007/s10562006-9003-7

(17) Faungnawakij, K.; Shimoda, N.; Fukunaga, T.; Kikuchi, R.; Eguchi, K. Appl. Catal. B: Environ. 2009, 92, 341. doi: 10.1016/ j.apcatb.2009.08.013

(18) Estrella, M.; Barrio, L.; Zhou, G.; Wang, X.; Wang, Q.; Wen, W.; Hanson, J. C.; Frenkel, A. I.; Rodriguez, J. A. J. Phys. Chem. C 2009, 113, 14411. doi: 10.1021/jp903818q

(19) Yang, X.; Wei, Y.; Su, Y.; Zhou, L. Fuel Process. Technol. 2010, 91, 1168. doi: 10.1016/j.fuproc.2010.03.032

(20) Andreev, A.; Idakiev, V.; Mihajlova, D.; Shopov, D. Applied Catalysis 1986, 22, 385. doi: 10.1016/S0166-9834(00)82645-7

(21) Idakiev, V.; Mihajlova, D.; Kunev, B.; Andreev, A. React. Kinet. Catal. L. 1987, 33, 119. doi: 10.1007/BF02066710

(22) Khan, A.; Chen, P.; Boolchand, P.; Smirniotis, P. G. J. Catal. 2008, 253, 91. doi: 10.1016/j.jcat.2007.10.018

(23) Reddy, G. K.; Smirniotis, P. G. Catal. Lett. 2011, 141, 27. doi: 10.1007/s10562-010-0465-2

(24) Lin, M. G.; Fang, K. G.; Li, D. B.; Sun, Y. H. Acta Phys. -Chim. Sin. 2008, 24 (5), 833. [林明桂, 房克功, 李德宝, 孙予罕. 物 理化学学报, 2008, 24 (5), 833.] doi: 10.3866/PKU. WHXB20080517

(25) Khan, A.; Smirniotis, P. G. J. Mol. Catal. A: Chem. 2008, 280, 43. doi: 10.1016/j.molcata.2007.10.022

(26) Yang, S. C.; Su, W. N.; Lin, S. D.; Rick, J.; Cheng, J. H.; Liu, J. Y.; Pan, C. J.; Liu, D. G.; Lee, J. F.; Chan, T. S.; Sheu, H. S.; Hwang, B. J. Appl. Catal. B: Environ. 2011, 106, 650. doi: 10.1016/j.apcatb.2011.06.030

(27) Kameoka, S.; Tanabe, T.; Tsai, A. P. Appl. Catal. A: Gen. 2010, 375, 163. doi: 10.1016/j.apcata.2009.12.035

(28) Faungnawakij, K.; Kikuchi, R.; Fukunaga, T.; Eguchi, K. Catal. Today 2008, 138, 157. doi: 10.1016/j.cattod.2008.05.004

(29) Strohmeier, B. R.; Levden, D. E.; Field, R. S.; Hercules, D. M. J. Catal. 1985, 94, 514. doi: 10.1016/0021-9517(85)90216-7

(30) Severino, F.; Brito, J. L.; Laine, J.; Fierro, J. L. G.; Agudo, A. L. J. Catal. 1998, 177, 82. doi: 10.1006/jcat.1998.2094 